ISSN 0103-9954

\title{
PRODUÇÃO DE MASSA SECA E EFICIÊNCIA NUTRICIONAL DE CLONES DE Eucalyptus dunnii E Eucalyptus benthamii EM FUNÇÃO DA ADIÇÃO DE DOSES DE FÓSFORO AO SOLO
}

\author{
DRY MATTER YIELD AND NUTRITIONAL EFFICIENCY OF Eucalyptus benthamii AND Eucalyptus \\ dunnii CLONES DUE TO ADDITION OF PHOSPHORUS RATES TO THE SOIL
}

\author{
James Stahl ${ }^{1}$ Paulo Roberto Ernani ${ }^{2}$ Luciano Colpo Gatiboni ${ }^{3}$ Djalma Miler Chaves ${ }^{4}$ \\ Cintia Urbano Neves ${ }^{5}$
}

\begin{abstract}
RESUMO
As espécies florestais diferem na capacidade de absorção, translocação, acúmulo e uso dos nutrientes. Estas diferenças ocorrem entre espécies, procedências, progênies, e mesmo entre clones de uma determinada espécie e influenciam o crescimento das mesmas. O objetivo deste experimento foi avaliar a produção de massa seca e a eficiência nutricional em relação ao fósforo de mudas de clone de Eucalyptus dunnii e de Eucalyptus benthamii cultivadas em vasos. O experimento foi conduzido por três meses, em casa de vegetação, num esquema fatorial $(2 \times 5)$, compreendendo as duas espécies e cinco doses de $\mathrm{P}(0,50,100$, 200 e $500 \mathrm{mg} \mathrm{dm}^{-3}$ ). Foram utilizadas amostras de um Cambissolo Húmico coletadas na camada 0-20 cm em reflorestamento de pinus, que apresentava $4,6 \mathrm{mg} \mathrm{dm}^{-3}$ de P. Aos 90 dias após o plantio, procedeu-se a coleta da biomassa da parte aérea e radicular das plantas, separadamente, para determinação da massa seca e do teor de P. A partir desses valores, determinou-se a eficiência de absorção (EAP), a eficiência de utilização de P (EUP) e a percentagem de recuperação do P aplicado pela biomassa. Os clones de Eucalyptus dunnii e Eucalyptus benthamii apresentaram aumentos quadráticos na produção de massa seca total e na eficiência de utilização de P com o aumento das doses de P. O Eucalyptus dunnii foi mais eficiente na produção de massa seca total assim como na utilização e recuperação de $\mathrm{P}$ em relação ao Eucalyptus benthamii, em todos os níveis de fornecimento de $\mathrm{P}$.
\end{abstract}

Palavras-chave: eucalipto; adubação fosfatada; nutrição florestal.

\begin{abstract}
Forest species differ in the absorption capacity, translocation, accumulation and use of nutrients. These differences occur between species, provenances, progenies, and even clones of specific species with influence in their growth. This study has as objective to evaluate the effect of phosphorus addition to the soil on the initial growth and nutritional efficiency of seedlings of Eucalyptus dunnii and Eucalyptus benthamii clones cultivated in pots. The experiment was conducted in a greenhouse for three months. Treatments consisted in a factorial design $(2 \times 5)$ with two species and five rates of $\mathrm{P}\left(0,50,100,200 \mathrm{e} 400 \mathrm{mg} \mathrm{dm}^{-3}\right)$. The soil is a Humic Haplumbrept collected from a 0-20 cm layer under a pine plantation, which had $4.6 \mathrm{mg} \mathrm{dm}^{-3} \mathrm{P}$. Ninety days after planting, shoots and roots biomass were harvested, dried at $65{ }^{\circ} \mathrm{C}$, weighed and analyzed for P. Subsequently, the absorption of $\mathrm{P}$, the use efficiency of $\mathrm{P}$ and the percentage of $\mathrm{P}$ applied recovered by the biomass were calculated. The clones of Eucalyptus dunnii and Eucalyptus benthamii showed a quadratic increase in total dry mass yield and in the use of $\mathrm{P}$ as the rate of $\mathrm{P}$ applied increased. Eucalyptus
\end{abstract}

1. Engenheiro Florestal, MSc. Pesquisador da Klabin S.A., Av. Araucária, s/n, CEP 84279-000, Telêmaco Borba (PR).jstahl@klabin.com.br

2. Engenheiro Agrônomo, PhD. Professor do Departamento de Solos, Universidade do Estado de Santa Catarina, Caixa Postal 281, CEP 88520-000, Lages (SC). Bolsista do CNPq. prernani@cav.udesc.br

3. Engenheiro Agrônomo, Dr. Professor do Departamento de Solos, Universidade do Estado de Santa Catarina, Caixa Postal 281, CEP 88520-000, Lages (SC). Bolsista do CNPq. gatiboni@cav.udesc.br

4. Engenheiro Agrônomo, PhD., Consultor da Klabin S.A, Av. Araucária, s/n, CEP 84279-000, Telêmaco Borba (PR).dm.chaves@terra.com.br

5. Engenheira Agrônoma, Mestranda em Manejo de Solos, Universidade do Estado de Santa Catarina, Caixa Postal 281, CEP 88520-000, Lages (SC). cintiaurb@hotmail.com

Recebido para publicação em 12/12/2010 e aceito em 5/04/2012 
dunnii was more efficient in terms of dry matter yield and use and recovery of $\mathrm{P}$ in relation to Eucalyptus benthamii, in all levels of added $\mathrm{P}$.

Keywords: eucalypt; phosphate fertilization; forest nutrition.

\section{INTRODUÇÃO}

As espécies florestais apresentam diferenças na capacidade de absorção, translocação, acúmulo e uso dos nutrientes, que influenciam o crescimento das mesmas. Estas diferenças ocorrem entre espécies, procedências, progênies, e mesmo entre clones de uma determinada espécie (GODOY e ROSADO, 2011). Guimarães (1993) relatou que genótipos que crescem bem em solos de baixa fertilidade devem ser selecionados, sendo desejáveis aqueles que tenham menores necessidades nutricionais, sem, contudo, comprometer a produtividade. Assim, o entendimento do grau de variabilidade genética sobre atributos nutricionais e fisiológicos do crescimento de plantas é fundamental para a seleção e o melhoramento mais eficientes e, consequentemente, para o aumento do ganho genético, com menor impacto sobre o nível de nutrientes do solo.

Para o gênero Eucalyptus, há variações nas eficiências de absorção e de utilização de um ou mais nutrientes entre espécies e entre clones de uma mesma espécie (SILVA, 1983; BARROS et al., 1985; MORAIS, 1990; GODOY e ROSADO, 2011). O fósforo (P) é o principal nutriente que confere caráter distinto às espécies de eucaliptos em relação ao crescimento e à eficiência nutricional (FURTINI NETO, 1994). A eficiência nutricional pode ser entendida de várias maneiras. Do ponto de vista agronômico, ela se refere à capacidade de um genótipo ter altas produções em um solo pobre em determinado nutriente, considerando-se um genótipo como padrão (GRAHAM, 1984). Sob o ponto de vista fisiológico, a eficiência nutricional refere-se à capacidade do genótipo de absorver o nutriente do solo, distribuí-lo e utilizá-lo internamente (GODDARD e HOLLIS, 1984).

Dependendo do nutriente, deve ser também considerada a eficiência de sua redistribuição interna (MOLL et al., 1982). Do ponto de vista nutricional, um genótipo eficiente é aquele que cresce e produz bem em condições de baixo suprimento de nutrientes pelo solo, e assim, comparativamente a outras cultivares, ter maior capacidade de absorver os nutrientes e utilizá-los na produção de biomassa (FURLANI et al., 1990). Entretanto, é muito difí- cil selecionar um material genético que apresente alta eficiência na utilização de todos os nutrientes essenciais. Sendo assim, a melhor estratégia é selecionar e utilizar materiais genéticos compatíveis com as características e necessidades dos sítios onde serão cultivados (CAMARGO et al., 2004).

A eficiência nutricional pode ser calculada pelo coeficiente de utilização biológico (CUB), que consiste na razão entre a massa seca (MS) e o acúmulo de nutrientes (Ac.N). Alternativamente, pode ser reescrito como o inverso do teor do nutriente, ou seja: $\mathrm{CUB}=\mathrm{MS} / \mathrm{Ac} . \mathrm{N}$, onde Ac. $\mathrm{N}=$ Teor $* \mathrm{MS}$, $\operatorname{logo}$ CUB $=1 /$ Teor. Entretanto, segundo Siddiqui e Glass (1981), a relação de eficiência nutricional deve sempre estar relacionada com a produção, para não incorrer em erro de selecionar cultivares com alta relação de eficiência nutricional e baixa produção. Para tanto, Siddiqui e Glass (1981) sugerem o índice de eficiência nutricional (EN). Este índice é calculado pela relação entre a MS da planta e a concentração do nutriente na mesma.

Souza (1994) menciona cinco características que contribuem para o aumento da eficiência na absorção e de utilização de nutrientes pelas plantas: adequada geometria e distribuição do sistema radicular, modificações químicas na rizosfera e exsudação de substâncias capazes de solubilizar nutrientes, presença de micorrizas, tolerância a condições de baixo pH ou aumento de teores de alumínio trocável, e taxa de absorção mais rápida em condições de baixas concentrações de nutrientes. Segundo Föhse et al. (1991), plantas com alta eficiência de absorção de $\mathrm{P}$ apresentam não só alta taxa de absorção do nutriente por unidade de massa de raiz (alto influxo), mas também alta relação parte aérea/raiz. A recuperação do $\mathrm{P}$ pelas plantas também pode variar devido à adsorção e baixa difusão do nutriente no solo. A formação de precipitados de fosfatos de ferro e de alumínio, em solos muito ácidos, também contribui para diminuição da disponibilidade de $\mathrm{P}$ as plantas (ERNANI et al., 2000).

O objetivo deste trabalho foi avaliar a produção de massa seca e a eficiência nutricional de mudas de clone de Eucalyptus dunnii e Eucalyptus benthamii cultivadas em um Cambissolo Húmico em resposta à adição de doses crescentes de fósforo. 


\section{MATERIAL E MÉTODOS}

O experimento foi conduzido em casa de vegetação, no viveiro de pesquisas da empresa Klabin S.A., em Otacílio Costa, SC, no período de outubro de 2008 a janeiro de 2009. Foi utilizado um Cambissolo Húmico alumínico léptico, coletado na camada de $0-20 \mathrm{~cm}$ em área de reflorestamento de Pinus taeda. $\mathrm{O}$ solo apresentava as seguintes características: $\mathrm{pH}-\mathrm{H}_{2} \mathrm{O}=3,9 ; \mathrm{Al}^{3+}=9,5 \mathrm{cmol}_{\mathrm{c}} \mathrm{dm}^{-3} ; \mathrm{Ca}=$ $0,6 \mathrm{cmol}_{\mathrm{c}} \mathrm{dm}^{3} ; \mathrm{Mg}=0,1 \mathrm{cmol}_{\mathrm{c}} \mathrm{dm}^{-3} ; \mathrm{P}=4,6 \mathrm{mg} \mathrm{dm}^{-3}$; $\mathrm{K}=50 \mathrm{mg} \mathrm{dm}{ }^{-3} ; 510 \mathrm{~g} \mathrm{~kg}^{-1}$ de argila e $34 \mathrm{~g} \mathrm{~kg}^{-1}$ de matéria orgânica (M.O). Posteriormente à coleta, 0 solo foi tamisado em peneira de $4 \mathrm{~mm}$, e em seguida recebeu 2,0 $\mathrm{g} \mathrm{kg}^{-1}$ de calcário dolomítico finamente moído, antes da aplicação dos tratamentos, com o objetivo de elevar a quantidade de $\mathrm{Ca}+\mathrm{Mg}$ para 2,0 $\mathrm{cmol}_{\mathrm{c}} \mathrm{dm}^{3}$.

Os tratamentos consistiram de um arranjo fatorial envolvendo duas espécies (Eucalyptus dunnii e Eucalyptus benthamii) e cinco doses de fósforo $\left(0,50,100,200\right.$ e $\left.400 \mathrm{mg} \mathrm{dm}^{-3}\right)$, com as quatro repetições arranjadas no delineamento experimental inteiramente casualizado. As doses de $\mathrm{P}$ foram selecionadas com base em trabalhos prévios desenvolvidos com $\mathrm{P}$ nos solos da região, em experimentos com vasos. A unidade experimental foi constituída por vaso plástico preenchido com 18 litros de solo no qual foi plantado uma muda. As doses de $\mathrm{P}$ foram homogeneizadas manualmente com todo o volume de solo de cada unidade experimental, no dia do plantio das mudas. Foram utilizadas mudas clonais homogêneas em altura e diâmetro, com três meses de idade, produzidas por propagação vegetativa no viveiro da Klabin, em Telêmaco Borba, PR.

Como fonte de fósforo, utilizou-se o superfosfato triplo $\left(46 \% \mathrm{P}_{2} \mathrm{O}_{5}\right)$ previamente tamizado em peneira com $2 \mathrm{~mm}$ de abertura. Em todas as unidades experimentais, foi realizada adubação básica logo após o plantio, via solução, de modo a fornecer as seguintes quantidades de nutrientes, em cada quilograma de solo seco: $50 \mathrm{mg}$ de $\mathrm{N}$ na forma de ureia $\left[\mathrm{CO}\left(\mathrm{NH}_{2}\right)_{2}\right], 100 \mathrm{mg}$ de $\mathrm{K}$ na forma de $\mathrm{KCl}, 5 \mathrm{mg}$ de $\mathrm{Zn}$ na forma de $\mathrm{ZnSO}_{4}$ e $5 \mathrm{mg}$ de $\mathrm{Cu}$ na forma de $\mathrm{CuSO}_{4}$. A umidade do solo foi mantida durante todo o período experimental em aproximadamente $80 \%$ da água retida na capacidade de campo. O volume de água evapotranspirado, obtido por meio de pesagem dos vasos, foi reposto diariamente.

Ao final de três meses procedeu-se a coleta manual total da massa da parte aérea e radicular das plantas, separadamente. A coleta das raízes foi efetuada por meio do peneiramento do solo e catação manual. A massa da parte aérea e das raízes foi seca a $65{ }^{\circ} \mathrm{C}$ em estufa com circulação de ar forçada, até atingir massa constante. Posteriormente à pesagem, realizou-se a moagem do material vegetal, em moinho tipo Wiley.

Após a moagem subamostras secas da parte aérea foram digeridas com ácido sulfúrico e água oxigenada, sem mistura de digestão, em bloco digestor previamente aquecido a $100{ }^{\circ} \mathrm{C}$. Procedeu-se o aumento gradual de temperatura até atingir $350{ }^{\circ} \mathrm{C}$. O P total das amostras foi determinado de acordo com a metodologia proposta por Murphy e Riley (1962). O fósforo no solo foi extraído pelo método de Mehlich-1, que é uma solução ácida, cuja concentração final possui $\mathrm{HCl}$ $0,05 \mathrm{~mol} \mathrm{~L}^{-1}$ e $_{2} \mathrm{SO}_{4} 0,0125 \mathrm{~mol} \mathrm{~L}^{-1}$, sendo a seguir determinado por colorimetria (TEDESCO et al., 1995).

A partir do teor de fósforo e da massa seca da parte aérea das plantas, determinou-se o $\mathrm{P}$ acumulado na massa da parte aérea, a eficiência de absorção de $\mathrm{P}$ (EAP = conteúdo de $\mathrm{P}$ na planta / massa seca da raiz) e a eficiência de utilização de $\mathrm{P}\left(\mathrm{EUP}=(\text { matéria seca })^{2} /\right.$ conteúdo de $\left.\mathrm{P}\right)$ de acordo com as metodologias propostas por Siddiqui e Glass (1981). Também foi determinada, para cada espécie, a percentagem de recuperação pela massa da parte aérea do $\mathrm{P}$ aplicado $[\% \mathrm{P}$ recuperado $=\mathrm{P}$ (conteúdo na planta fertilizada) - $\mathrm{P}$ (conteúdo planta testemunha)*100 / P (quantidade de aplicada)].

Os dados obtidos foram submetidos à análise de variância (teste $\mathrm{F}$ ) para avaliar a significância dos efeitos dos fatores (doses de $\mathrm{P}$ e espécies) e da interação entre eles. Para os parâmetros de crescimento (massa seca da parte aérea, radicular e total) e para os parâmetros de eficiência nutricional (teor de $\mathrm{P}$ na massa da parte aérea, $\mathrm{P}$ acumulado, eficiência de absorção de $\mathrm{P}$ e eficiência de utilização de P) foram ajustadas equações de regressão com uso do pacote estatístico SAS (2007).

\section{RESULTADOS E DISCUSSÃO}

\section{Produção de Massa Seca}

As doses de $\mathrm{P}$ influenciaram a produção de massa seca da parte aérea, das raízes e total de maneira diferente entre as espécies de eucalipto (Eucalyptus dunnii e Eucalyptus benthamii) $(\mathrm{P}<0,01)$ (Tabela 1). 
TABELA 1: Análise de variância com valores de quadrado médio (QM) e coeficiente de variação da massa seca da raiz (MsRA), da massa seca da parte aérea (MsPA) e da massa seca total (MsTO) de Eucalyptus dunnii e Eucalyptus benthamii, aos três meses após o plantio, em função de doses de fósforo adicionadas ao solo.

TABLE 1: Analysis of variance with mean square and coefficient of variance for root, shoot and total dry matter of Eucalyptus dunnii and Eucalyptus benthamii, three months after planting as influenced by increasing rates of phosphorus added to the soil.

\begin{tabular}{cccc}
\hline \multirow{2}{*}{$\begin{array}{c}\text { Fonte de } \\
\text { variação }\end{array}$} & \multicolumn{3}{c}{ QM } \\
\cline { 2 - 4 } & MsRA & MsPA & MsTO \\
\hline & $\left(\mathrm{g} \mathrm{vaso}^{-1}\right)$ & $\left(\mathrm{g} \mathrm{vaso}^{-1}\right)$ & $\left(\mathrm{g} \mathrm{vaso}^{-1}\right)$ \\
Espécie (E) & $1339,9^{* *}$ & $789,8^{* *}$ & $29863,5^{* *}$ \\
Doses P (P) & $357,4^{* *}$ & $293,3^{* *}$ & $10066,6^{* *}$ \\
$\mathrm{E} * \mathrm{P}$ & $89,2^{* *}$ & $30,4^{* *}$ & $1255,2^{* *}$ \\
\hline $\mathrm{CV}(\%)$ & 13,9 & 6,7 & 7,7 \\
\hline
\end{tabular}

Em que: $* *=$ significativo $(\mathrm{P}<0,01)$.

O rendimento de massa seca da parte aérea (MsPA) das duas espécies apresentou um comportamento quadrático com o aumento das doses de $\mathrm{P}$ adicionadas ao solo. Em todos os níveis de $\mathrm{P}$, o Eucalyptus dunnii produziu mais massa seca aérea do que Eucalyptus benthamii, sendo que no tratamento onde não foi aplicado fósforo a produção foi 2,26 vezes maior, evidenciando uma melhor adaptabilidade do Eucalyptus dunnii em solos com baixa disponibilidade de P (Figura 1). Eficiência de utilização e resposta diferencial de $\mathrm{P}$ por espécies de eucalipto têm sido observadas por vários autores em diferentes condições (FURTINI NETO, 1994; GONÇALVES e PASSOS, 2000; PINTO et al., 2011). Furtini Neto (1994) avaliou a resposta de mudas de cinco espécies de eucalipto ao fornecimento de $\mathrm{P}$ e também encontrou comportamento quadrático na produção de biomassa, com máximas respostas obtidas para doses variando de 250 a $350 \mathrm{mg} \mathrm{dm}^{-3}$ de $P$.

A interação entre as doses de $\mathrm{P}$ e as espécies de eucalipto foi significativa $(\mathrm{P}<0,01)$, confirmando a diferença de comportamento na produção de massa da parte aérea (Tabela 1). A produção de massa seca do Eucalyptus dunnii foi maior do que a do Eucalyptus benthamii em todas as doses de P, demonstrando uma melhor resposta ao fornecimento de P. A máxima produção por vaso foi de $134 \mathrm{~g}$, obtida na dose estimada de $256 \mathrm{mg} \mathrm{dm}^{-3} \mathrm{de} \mathrm{P}$.

$\mathrm{O}$ efeito das doses de $\mathrm{P}$ aplicadas ao solo na produção de raízes também variou com a espécie de eucalipto (Tabela 1; Figura 2). A produção de massa seca das raízes (MsRA) do Eucalyptus benthamii aumentou linearmente com o aumento das doses de P (Figura 2); a do Eucalyptus dunnii, entretanto, aumentou de forma quadrática, apresentando a produção máxima de $34 \mathrm{~g} \mathrm{vaso}^{-1} \mathrm{com}$ a adição de $251 \mathrm{mg} \mathrm{dm}^{-3}$ de P. À semelhança do que ocorreu com a produção de massa seca da parte aérea, a massa seca radicular estimada também foi maior para o Eucalyptus dunnii em relação ao Eucalyptus benthamii, em todas as doses de $\mathrm{P}$, sendo 1,7 e 2,0 vezes maior nas doses de 0 e $300 \mathrm{mg} \mathrm{dm}^{-3}$, respectivamente. Efeito diferencial do $\mathrm{P}$ adicionado ao solo no rendimento de diferentes partes da planta de eucalipto também foram encontradas por Gonçalves e Passos (2000). Eles avaliaram o efeito de doses de $\mathrm{P}$ e de déficit hídrico em espécies de eucalipto (Eucalyptus pellita, Eucalyptus camaldulensis, Eucalyptus citriodora, Eucalyptus cloeziana e Eucalyptus urophylla) e verificaram que o Eucalyptus camaldulensis foi a espécie que teve o

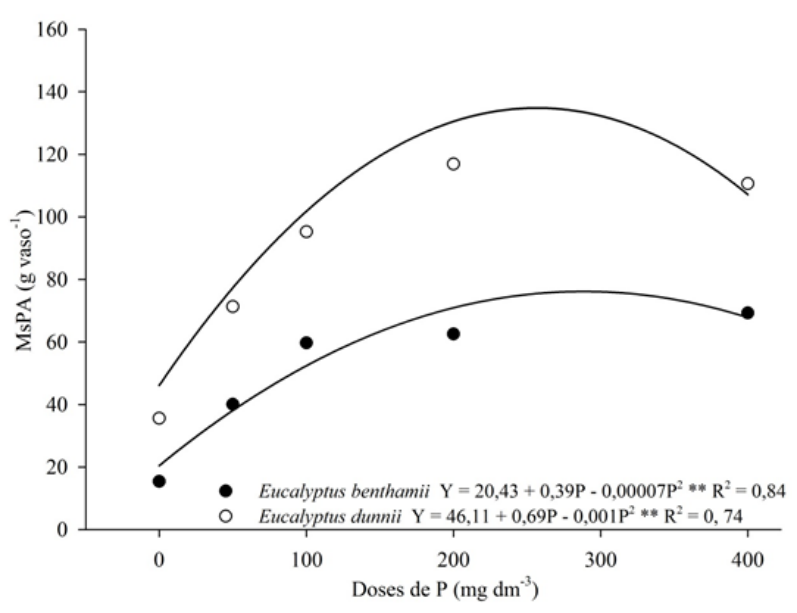

FIGURA 1: Produção de massa seca da parte aérea de Eucalyptus dunnii e Eucalyptus benthamii, aos três meses após o plantio, em função de doses de fósforo adicionadas ao solo.

FIGURE 1: Shoot dry matter of Eucalyptus dunnii and Eucalyptus benthamii, three months after planting as influenced by increasing rates of phosphorus added to the soil. 


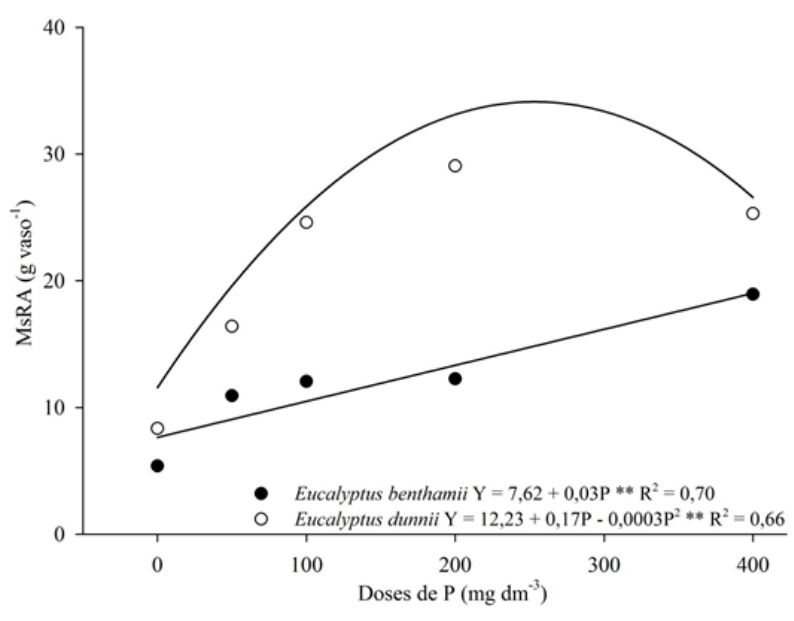

FIGURA 2: Produção de massa seca radicular de Eucalyptus dunnii e Eucalyptus benthamii, aos três meses após o plantio, em função de doses de fósforo adicionadas ao solo.

FIGURE 2: Root dry matter production of Eucalyptus dunnii and Eucalyptus benthamii, three months after planting as influenced by increasing rates of phosphorus added to the soil.

maior aumento na relação raiz/parte aérea e a menor redução na produção de matéria seca total, no tratamento que tinha déficit hídrico e o menor nível de $\mathrm{P}$.

Visualmente foi possível verificar diferenças na morfologia das raízes entre as duas espécies, tendo o Eucalyptus dunnii apresentado um sistema radicular bem mais ramificado e com raízes mais finas do que o Eucalyptus benthamii. Sabe-se que a geometria das raízes influencia o crescimento da planta e a aquisição de nutrientes, especialmente daqueles com baixa mobilidade no solo, a exemplo do P. Por isso, plantas com sistema radicular mais fino e fasciculado exploram um maior volume de solo, o que facilita a aquisição do nutriente, principalmente sob condições de limitada disponibilidade no solo (FÖSHE et al., 1991). A importância do efeito do sistema radicular na absorção e uso de P por plantas de eucalipto pode ser verificada nos dados obtidos por Ribeiro et al. (2010). Eles verificaram, em experimento de vasos conduzidos com Latossolos de Minas Gerais, que o aumento da densidade do solo diminuiu o rendimento de massa seca produzida pelo Eucalyptus grandis, por afetar negativamente o desenvolvimento do sistema radicular, porém, esse decréscimo foi revertido pelo aumento da dose de $\mathrm{P}$ aplicada.

A produção de massa seca total seguiu o mesmo comportamento quadrático verificado para massa seca da parte aérea, sempre com o Eucalyptus dunnii (Figura 3) apresentando a maior produção de massa total, em todas as doses testadas. As máximas produções de massa seca total (MsTO) para Eucalyptus dunnii e para Eucalyptus benthamii foram, respectivamente, de $168 \mathrm{~g} \mathrm{vaso}^{-1}$ na dose estimada de $256 \mathrm{mg} \mathrm{dm}^{-3}$ de $\mathrm{P}$ e de $92 \mathrm{~g} \mathrm{vaso}^{-1}$ na dose de $310 \mathrm{mg} \mathrm{dm}^{-3}$ de P (Figura 3). Verificou-se, portanto, maior resposta do Eucalyptus dunnii à adição de $\mathrm{P}$, já que são necessárias $2,3 \mathrm{mg} \mathrm{dm}^{-3}$ de $\mathrm{P}$ para $\mathrm{o}$ incremento de $1,0 \mathrm{~g}$ vaso $^{-1}$ de massa total, enquanto que para o Eucalyptus benthamii são necessárias $4,8 \mathrm{mg} \mathrm{dm}^{-3}$ de $\mathrm{P}$ para produzir o mesmo incremento. A resposta em produção de massa seca até doses altas de P pode ser explicada pela condução das plantas em vasos, onde há uma maior relação raízes/solo relativamente às plantas cultivadas no campo. Em vasos, o sistema radicular explora mais intensamente o pequeno volume de solo existente, pois há uma sobreposição das zonas de depleção de $\mathrm{P}$ ao redor das raízes, o que dificilmente acontece no campo. D'Avila (2008) testou a adição de $\mathrm{P}$ a substratos, em quantidades de 0 a $1046 \mathrm{mg} \mathrm{dm}^{-3} \mathrm{e}$ verificou que doses de 400 a $490 \mathrm{mg} \mathrm{dm}^{-3}$ proporcionaram os melhores resultados na produção de mudas de três clones de Eucalyptus urograndis. Estudando o efeito da

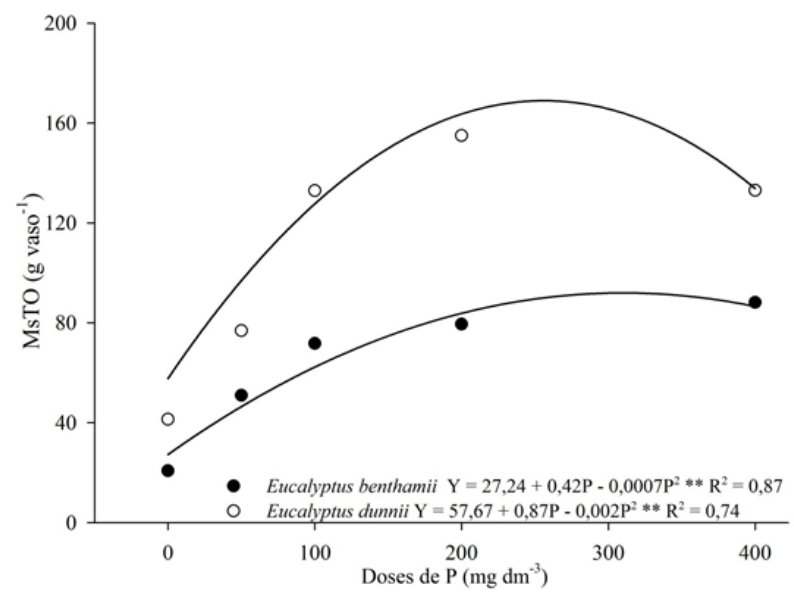

FIGURA 3: Produção de massa seca total de Eucalyptus dunnii e Eucalyptus benthamii, aos três meses após o plantio, em função de doses de fósforo adicionadas ao solo.

FIGURE 3: Total dry matter of Eucalyptus dunnii and Eucalyptus benthamii, three months after planting as influenced by increasing rates of phosphorus added to the soil. 
adição de doses e da localização de $\mathrm{P}$ a Latossolos de Minas Gerais, Leite et al. (2009) verificaram que o rendimento de massa seca da parte aérea do eucalipto teve baixa relação com a quantidade de $\mathrm{P}$ aplicada por vaso, porém, tanto a massa seca quanto o crescimento inicial das plantas foram altamente dependentes da dose de $\mathrm{P}$ aplicada na cova, junto à planta.

Para a dose correspondente a $90 \%$ da produção máxima estimada, o Eucalyptus dunnii apresentou produção relativa de massa seca total de 47 pontos percentuais maiores do que o Eucalyptus benthamii, com uma dose $22 \%$ menor (Tabela 2), confirmando a sua melhor resposta ao fornecimento de $\mathrm{P}$ e consequente maior produção de massa seca total.

\section{Parâmetros de eficiência nutricional}

Todos os parâmetros relacionados com a eficiência nutricional de $\mathrm{P}$ diferiram entre as duas espécies de eucalipto ao nível de $1 \%$ de significância $(\mathrm{P}<0,01)$ (Tabela 3$)$. A interação entre espécies e doses de $\mathrm{P}$ foi estatisticamente significativa $(\mathrm{P}<0,01)$ para o $\mathrm{P}$ acumulado na massa da parte aérea e para a eficiência de absorção do nutriente.

$\mathrm{O}$ conteúdo de $\mathrm{P}$ acumulado na massa seca da parte aérea seguiu o mesmo comportamento quadrático encontrado para a produção de massa seca total. Mesmo as concentrações de P tendo sido menores no Eucalyptus dunnii, este apresentou os maiores conteúdos do nutriente acumulado, em todas as doses testadas (Figura 4), em virtude da maior produção de massa seca produzida por esta

TABELA 2: Massa seca total e dose de fósforo necessária para produzir $90 \%$ da massa seca máxima estimada para as espécies de Eucalyptus dunnii e Eucalyptus benthamii, aos três meses após o plantio.

TABLE 2: Total dry matter and concentration of phosphorus needed to produce $90 \%$ of maximum estimated dry matter for the Eucalyptus dunnii and Eucalyptus benthamii, three months after planting.

\begin{tabular}{lccc}
\hline Espécie & $\begin{array}{c}\text { Massa } \\
\text { seca total }\end{array}$ & $\begin{array}{c}\text { Produção } \\
\text { relativa }\end{array}$ & $\begin{array}{c}\text { Doses } \\
\text { de P }\end{array}$ \\
\hline & $\left(\mathrm{g} \mathrm{vaso}^{-1}\right)$ & $(\%)$ & $\left(\mathrm{mg} \mathrm{dm}^{-3}\right)$ \\
Eucalyptus dunnii & 156,0 & 100 & 151,9 \\
Eucalyptus benthamii & 82,8 & 53 & 193,2 \\
\hline
\end{tabular}

espécie relativamente à produzida pelo Eucalyptus benthamii.

A quantidade de $\mathrm{P}$ no solo, ao final dos três meses de condução do experimento, aumentou linearmente com o aumento da dose de $\mathrm{P}$ aplicada, porém, com diferenças entre as duas espécies (Figura 5). Mesmo o Eucalyptus dunnii tendo acumulado mais $\mathrm{P}$ na biomassa, o $\mathrm{P}$ do solo cultivado com Eucalyptus dunnii foi $25 \%$ maior do que naquele cultivado com Eucalyptus benthamii, em todas as doses testadas. Este fato demonstra que o Eucalyptus dunnii pode ter utilizado quantidades de $\mathrm{P}$ de formas não detectadas pelo método de extração do P solo.

A eficiência de absorção de P (EAP) também foi distinta entre as duas espécies (Figura 6). O Eucalyptus dunnii obteve ganhos lineares crescentes na eficiência de absorção à medida que aumentou o fornecimento desse nutriente, partindo de $10,5 \mathrm{mg} \mathrm{P} \mathrm{g}^{-1}$, na dose zero, e atingindo eficiência

TABELA 3: Análise de variância com valores de quadrado médio e coeficiente de variação do teor de fósforo na massa seca $(\mathrm{P})$, fósforo acumulado na massa seca (PAc.), eficiência de absorção de fósforo(EAP) e eficiência de utilização de fósforo (EUP) de Eucalyptus dunnii e Eucalyptus benthamii, aos três meses após o plantio, em função de doses de fósforo adicionadas ao solo.

TABLE 3: Analysis of variance with mean square and coefficient of variance of phosphorus concentration and accumulated in the dry matter, absorption and efficiency use of phosphorus by Eucalyptus dunnii and Eucalyptus benthamii, three months after planting as influenced by increasing rates of phosphorus added to the soil.

\begin{tabular}{ccccc}
\hline \multirow{2}{*}{$\begin{array}{c}\text { Fonte de } \\
\text { variação }\end{array}$} & $\mathrm{P}$ & PAc. & EAP & EUP \\
\cline { 2 - 5 } & $(\%)$ & $\left(\mathrm{mg} \mathrm{vaso}^{-1}\right)$ & $\left(\mathrm{mgP} \mathrm{g}^{-1}\right)$ & $\left(\mathrm{mg}^{2} \mathrm{~ms} \mathrm{mg}^{-1} \mathrm{P}\right)$ \\
& & $4,21^{* *}$ \\
Espécie (E) & $0,024^{* *}$ & $140,89 *$ & $99,26 *$ & $2,26^{* *}$ \\
Doses P (P) & $0,013^{*}$ & $107,72^{* *}$ & $80,31^{*}$ & $4,47^{* *}$ \\
$\mathrm{E} * \mathrm{P}$ & $0,001^{\mathrm{ns}}$ & $6,34^{* *}$ & $30,67^{\mathrm{ns}}$ & $7,96 * *$ \\
\hline $\mathrm{CV}(\%)$ & 11,18 & 12,82 & 26,57 & 11,50 \\
\hline
\end{tabular}

Em que: ${ }^{\text {ns }}=$ não significativo; $*=$ significativo $(\mathrm{P}<0,05)$; $* *=$ significativo $(\mathrm{P}<0,01)$. 


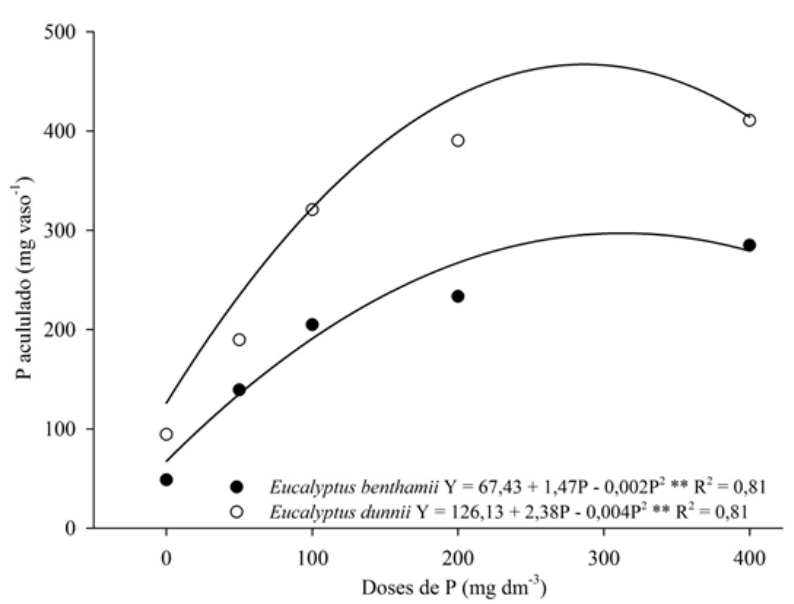

FIGURA 4: Conteúdo de P acumulado na de massa da parte aérea de Eucalyptus dunnii e Eucalyptus benthamii, aos três meses após o plantio, em função de doses de fósforo adicionadas ao solo.

FIGURE 4: Content of phosphorus accumulated in the shoots of Eucalyptus dunnii and Eucalyptus benthamii, three months after planting as influenced by increasing rates of phosphorus added to the soil.

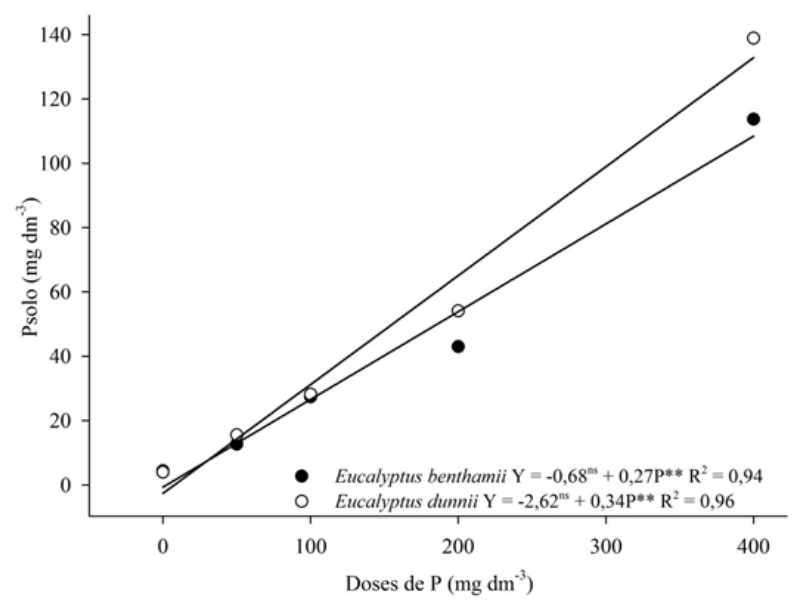

FIGURA 5: Valores de P disponível no solo, aos três meses após o plantio de Eucalyptus dunnii e Eucalyptus benthamii, em função de doses de fósforo adicionadas ao solo.

FIGURE 5: Concentrations of phosphorus available in the soil three months after planting Eucalyptus dunnii and Eucalyptus benthamii as influenced by increasing rates of phosphorus added to the soil.

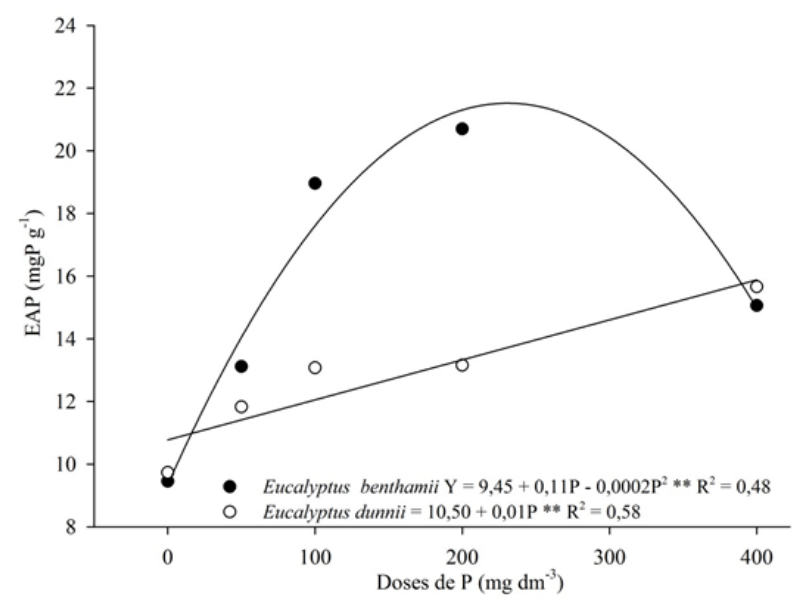

FIGURA 6: Eficiência de absorção de $\mathrm{P}$ de Eucalyptus dunnii e Eucalyptus benthamii, aos três meses após o plantio, em função de doses de fósforo adicionadas ao solo.

FIGURE 6: Efficiency phosphorus absorption by Eucalyptus dunnii and Eucalyptus benthamii, three months after planting as influenced by increasing rates of phosphorus added to the soil.

máxima de 14,3 $\mathrm{mg} \mathrm{P} \mathrm{g}^{-1}$ na dose de $400 \mathrm{mg} \mathrm{dm}^{-3} \mathrm{de}$ P. Por outro lado, o Eucalyptus benthamii apresentou ganhos crescentes até um máximo de eficiência de absorção de $21,9 \mathrm{mg} \mathrm{P} \mathrm{g}^{-1}$ para a dose estimada de $228 \mathrm{mg} \mathrm{dm}^{-3}$; após isso, houve redução até $14,9 \mathrm{mg} \mathrm{P}$ $\mathrm{g}^{-1}$ para a dose $400 \mathrm{mg} \mathrm{dm}^{-3}$. Essa diferença de comportamento das espécies pode ser explicada em função do crescimento radicular das espécies, que foi linear para o Eucalyptus benthamii e quadrático para o Eucalyptus dunnii, uma vez que a EAP é calculada pelo quociente entre o conteúdo de $\mathrm{P}$ na planta e a massa seca do sistema radicular.

O comportamento da eficiência de utilização de P não diferiu entre as espécies com a variação na disponibilidade de fósforo no solo. A eficiência de utilização de $\mathrm{P}$ aumentou até níveis máximos de produção de massa seca nas doses intermediárias, decrescendo nas maiores doses (Figura 7). Considerando que as curvas de crescimento e atuação enzimática são hipérboles retangulares, este comportamento era esperado, corroborando resultados de Siddiqui e Glass (1981) em estudo realizado com potássio. Por outro lado, o Eucalyptus dunnii demonstrou maior eficiência de utilização de $\mathrm{P}$ do que o Eucalyptus benthamii, em todas as doses de $\mathrm{P}$, com máxima eficiência de utilização $\left(39,5 \mathrm{~g}^{2} \mathrm{~ms} \mathrm{mg}^{-1}\right)$ para a 
dose estimada de $233 \mathrm{mg} \mathrm{dm}^{-3}$. Para Eucalyptus benthamii, a máxima eficiência $\left(19,9 \mathrm{~g}^{2} \mathrm{~ms} \mathrm{mg}^{-1}\right)$ de $P$ foi obtida na dose de $269 \mathrm{mg} \mathrm{dm}^{-3}$, representando um decréscimo de 49,7\% em relação ao Eucalyptus dunnii. No tratamento onde não foi adicionado $\mathrm{P}$, a eficiência do Eucalyptus dunnii na utilização e conversão em biomassa foi a maior, demonstrando sua melhor habilidade em se adaptar a ambientes com baixos níveis deste nutriente. À medida que aumenta a disponibilidade de $\mathrm{P}$ no solo até os níveis ideais para as máximas produções, ocorre também maior resposta do Eucalyptus dunnii na utilização de $\mathrm{P}$ em relação ao Eucalyptus benthamii. Diferenças na eficiência de utilização de $\mathrm{P}$ entre espécies de eucalipto também foram verificadas por outros autores. Alvarez et al. (2006) verificaram que híbridos de Eucalyptus urophylla $x$ Eucalyptus grandis usaram o P do solo mais eficientemente do que qualquer das duas espécies. Pinto et al. (2011) avaliaram seis clones de eucalipto em experimento de solução nutritiva e verificaram que eles diferiram tanto na produção de biomassa como na eficiência nutricional, avaliada pela absorção, translocação e utilização dos nutrientes.

A quantidade de $\mathrm{P}$ recuperada na massa da parte aérea diminuiu para as duas espécies com o aumento das doses de P (Tabela 4). O Eucalyptus

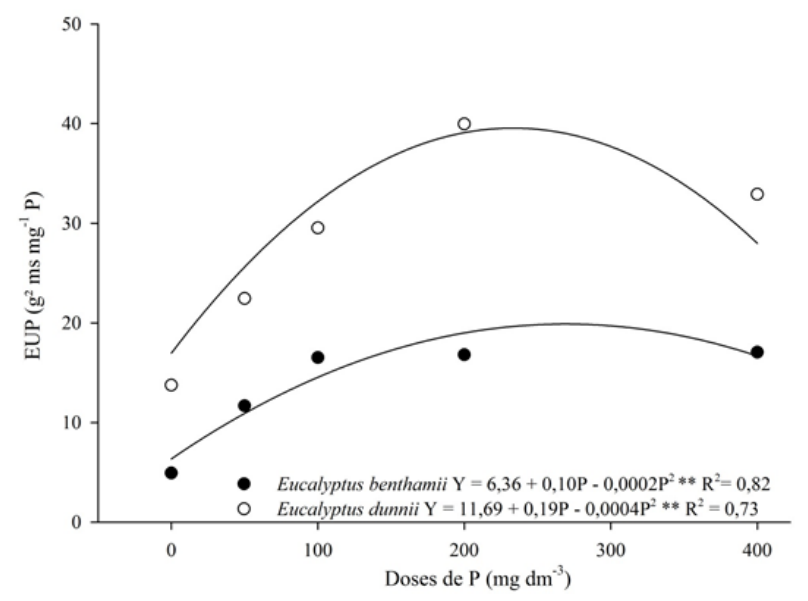

FIGURA 7: Eficiência de utilização de $\mathrm{P}$ de Eucalyptus dunnii e Eucalyptus benthamii, aos três meses após o plantio, em função de doses de fósforo adicionadas ao solo.

FIGURE 7: Efficiency phosphorus use by Eucalyptus dunnii and Eucalyptus benthamii, three months after planting as influenced by increasing rates of phosphorus added to the soil. dunnii apresentou uma maior percentagem de recuperação do que o Eucalyptus benthamii, em todas as doses, sendo 1,7 e 1,5 vezes maior nas doses de 100 e $400 \mathrm{mg} \mathrm{dm}^{-3}$ de $\mathrm{P}$, respectivamente. Diminuição da eficiência de utilização de fósforo pelas plantas com o aumento da dose aplicada tem sido verificada na literatura (FAGERIA et al., 2011).

Este melhor aproveitamento, em todas as doses do fertilizante fosfatado adicionado pelo Eucalyptus dunnii, corrobora com a maior produção de massa seca total, confirmando sua maior eficiência de utilização do nutriente relativamente ao Eucalyptus benthamii.

TABELA 4: Recuperação de fósforo na massa da parte aérea de Eucalyptus dunnii e Eucalyptus benthamii, aos três meses após o plantio, em função de doses de fósforo adicionadas ao solo.

TABLE 4: Phosphorus recovered by total dry matter of Eucalyptus dunnii and Eucalyptus benthamii, three months after planting as influenced by increasing rates of phosphorus added to the soil.

\begin{tabular}{|c|c|c|c|}
\hline \multirow{2}{*}{ Espécie } & \multicolumn{3}{|c|}{$\mathrm{P}\left(\mathrm{mg} \mathrm{dm}^{-3}\right)$} \\
\hline & 100 & 200 & 400 \\
\hline & ----------- & $\%$ & ----- \\
\hline Eucalyptus dunnii & 16,27 & 8,59 & 4,95 \\
\hline Eucalyptus benthamii & 9,43 & 5,13 & 3,28 \\
\hline
\end{tabular}

\section{CONCLUSÃo}

A adição de $\mathrm{P}$ ao solo aumentou a produção de massa seca e interferiu nos parâmetros relacionados com a eficiência nutricional de mudas de clones de Eucalyptus dunnii e Eucalyptus benthamii cultivados em vasos. A recuperação de $\mathrm{P}$ na massa da parte aérea das plantas diminuiu para as duas espécies com o aumento das doses de P. O Eucalyptus dunnii foi mais eficiente do que o Eucalyptus benthamii na produção de massa seca aérea e radicular, assim como na utilização de $\mathrm{P}$, em todas as doses de P aplicadas.

\section{REFERÊNCIAS BIBLIOGRÁFICAS}

ALVAREZ, V. F. C. et al. Improving phosphorus availability from Patos phosphate rock for eucalyptus: A study with $32 \mathrm{P}$ radiotracer. Scientia Agricola, v. 63, p. 65-69, 2006. 
BARROS, N. F. et al. Biomassa, absorção e eficiência de utilização de nutrientes por clones de eucalipto na região norte do Espírito Santo. In: CONGRESSO BRASILEIRO DE CIÊNCIA DO SOLO, 20., Campinas, 1985. Programa e resumos. Campinas: SBCS, 1985. p.109

CAMARGO, M. L. P. et al. Considerações sobre eficiência nutricional em Eucalyptus. Científica, Jaboticabal, v. 32, n. 2, p. 191-196, 2004.

D'AVILA, F. S. Efeito do fósforo, nitrogênio e potássio na produção de mudas clonais de eucalipto. 2008. 53 f. Dissertação (Mestrado em Ciência Florestal) - Universidade Federal de Viçosa, Viçosa, 2008.

ERNANI, P. R. et al. Influência da combinação de fósforo e calcário no rendimento de milho. Revista Brasileira de Ciência do Solo, v. 24, p. 537-544, 2000.

FAGERIA, N. K. et al. Lowland rice genotypes evaluation for phosphorus use efficiency in tropical lowland. Journal of Plant Nutrition, v. 34, n. 8, p. 1087-1095, 2011.

FÖSHE, D. et al. Phosphorus efficiency of plants: II. Significance of root radius, root hairs and catioanion balance for phosphorus influx in seven plant species. Plant and Soil, v. 132, p. $261-272,1991$. FURLANI, A. M. C.; USBERTI FILHO, J. A. Capimcolonião: eficiência na absorção e na utilização de fósforo em solução nutritiva. Bragantia, Campinas, v. 49 , n. 2 , p. $413-423.1990$.

FURTINI NETO, A. E. Eficiência nutricional, cinética de absorção e frações fosfatadas em Eucalyptus spp. 1994. 99 f. Tese (Doutorado em Solos e Nutrição de Plantas) - Universidade Federal de Viçosa, Viçosa, 1994.

GODDARD, R. E.; HOLLIS, C. A. The genetic basis of forest tree nutrition. In: BOWEN, G. D.; NANBIAR, E.K.S. (Ed.) Nutrition of plantation forest. London: Academic Press, 1984. p. 237-258. GODOY, T. G.; ROSADO, S. C. S. Efficiency of phosphorus use in young plants of Eucalyptus urophylla S. T. Blake. Cerne, Lavras, v. 17, n. 3, p. 303-308, 2011.

GONÇALVES, M. R.; PASSOS, C. A. M. Crescimento de cinco espécies de eucalipto submetidas a déficit hídrico em dois níveis de fósforo. Ciência Florestal, v. 10, n. 2, p. 145-161 $145,2000$.

GRAHAM, R. D. Breeding for nutritional characteristics in cerels. In: TINKER, P. B.; LAUCHLI,A. (Ed.) Advances in plant nutrition. New York: Praeger, 1984. p. 57-102.
GUIMARÃES, H. S. Variabilidade genética para eficiência nutricional em progênies de Eucalyptus camaldulensis e Eucalyptus citriodora. 1993. 68 f. Dissertação (Mestrado em Ciência Florestal) Universidade Federal de Viçosa. Viçosa, 1993.

LEITE, P. B. et al. Níveis críticos de fósforo, para eucalipto, em casa de vegetação, em função da sua localização no solo. Revista Brasileira de Ciência do Solo, Viçosa, v. 33, p. 1311-1322, 2009.

MOLL, R. H. et al. Analysis and interpretation of factors which contribute to efficiency of nitrogen utilization. Agronomy Journal, v. 74, p. 562-564, 1982.

MORAIS, E. J. et al. Biomassa e eficiência nutricional de espécies de eucalipto em duas regiões bioclimáticas de Minas Gerais. Revista Brasileira de Ciência do Solo, Campinas, v. 14, n. 3, p. 353362, 1990.

MURPHY, J.; RILEY, J. P. A modified single solution method for the determination of phosphate in natural waters. Analytica Chemical Acta, v. 27, p. 31-36, 1962.

PINTO, S. I. C. et al. Eficiência nutricional de clones de eucalipto na fase de mudas cultivados em solução nutritiva. Revista Brasileira de Ciência do Solo, Viçosa, v. .35 n. 2, p. 523-533, 2011.

RIBEIRO, M. A. V. et al. Resposta da soja e do eucalipto ao aumento da densidade do solo e a doses de fósforo. Revista Brasileira de Ciência do Solo, Viçosa, v. 34, n. 4, p. 1157-1164, 2010.

SAS. 2007. SAS 9.1.3 (TS1M3) for Windows Microsoft. SAS Institute Inc., Cary, USA, sem paginação.

SIDDIQUI, M. Y.; GLASS, A. D. M. Utilization index: a modified approach to the estimation and comparison of nutrient utilization efficiency in plants. Journal Plant Nutrition, v. 4, p. 289-302, 1981.

SILVA, H. D. et al Biomassa, concentração e conteúdo de nutrientes em cinco espécies de Eucalyptus plantadas em solos de baixa fertilidade.

Boletim de Pesquisa Florestal, n.6/7, p. 9-25, 1983. SOUZA, M. E. Correlação adulto-juvenil para eficiência nutricional e comportamento de clones de Eucalyptus grandis em dois níveis de fertilidade do solo. 1994. 49 f. Dissertação (Mestrado em Ciência Florestal) - Universidade Federal de Viçosa, Viçosa, 1994.

TEDESCO, M. J. et al. Análise de solo, plantas e outros materiais. 2. ed. Porto Alegre, Universidade Federal do Rio Grande do Sul, 1995. 147 p. (Boletim Técnico, 5). 